\title{
Activity in the Posterior Parietal Cortex Mediates Visual Dominance over Kinesthesia
}

\author{
Nobuhiro Hagura, ${ }^{1,2}$ Tomohiko Takei, ${ }^{1}$ Satoshi Hirose, ${ }^{1,3}$ Yu Aramaki, ${ }^{4,6}$ Michikazu Matsumura, ${ }^{1}$ Norihiro Sadato,${ }^{4,5}$ \\ and Eiichi Naito ${ }^{1,3,6}$ \\ ${ }^{1}$ Graduate School of Human and Environmental Studies, Kyoto University, Kyoto 606-8501, Japan, ${ }^{2}$ The Japan Society for the Promotion of Science, Tokyo \\ 102-8472, Japan, ${ }^{3}$ Advanced Telecommunications Research Institute International Computational Neuroscience Laboratories, Kyoto 619-0288, Japan, \\ ${ }^{4}$ National Institute for Physiological Sciences, Okazaki 444-8585, Japan, ${ }^{5}$ Japan Science and Technology Corporation/Research Institute of Science and \\ Technology for Society, Kawaguchi 332-0012, Japan, and ${ }^{6}$ National Institute of Information and Communication Technology (ICT), Research Department \\ 1, Kobe Advanced ICT Research Center, Biophysical ICT Group, Kyoto 619-0288, Japan
}

When both visual and kinesthetic information of a limb are available, vision is usually the dominant source of information used to perceive the spatial location. In this study, we conducted behavioral and functional magnetic resonance imaging (fMRI) experiments to examine the brain mechanisms underlying the visual dominance over kinesthesia in perceiving the position of a hand. We used tendon vibration to induce an illusory percept of flexion movement of an immobile hand, while the participants viewed a live image of either the vibrated or nonvibrated static hand through an on-line video camera. The intensity of illusory movement was significantly attenuated (for both the left and right hands) only when the participants viewed the static image of the vibrated hand. The fMRI study showed that the posterior parietal cortex (PPC) is specifically involved in the attenuation of illusory movement and that the activity of the PPC was associated with the degree of attenuation. This indicates that PPC is involved in the multisensory processing that occurs when vision overrules simultaneously available kinesthetic information for estimating the spatial location of a limb. It is thus suggested that the human parietal cortex may play a critical role in the maintenance of a coherent body image when the brain receives potentially conflicting multisensory information from the body.

Key words: visual dominance over kinesthesia; bodily perception; posterior parietal cortex; tendon vibration; functional magnetic resonance imaging (fMRI); multisensory

\section{Introduction}

Humans can perceive their body location in space based on both visual and kinesthetic/proprioceptive information (Head and Holmes, 1911; Graziano and Gross, 1998). In the absence of visual information, kinesthetic/proprioceptive information contributes to perception of the spatial location of a limb (Naito, 2004). However, somatosensory receptors, such as muscle spindle receptors, do not provide information that directly specifies the absolute location (Burgess et al., 1982). Thus, when visual information is available, the limb position is estimated, based mainly on the visual information (Smeets et al., 2006). This phenomenon is generally referred to as "visual dominance over proprioception/kinesthesia" (van Beers et al., 1996, 1998; Botvinick

\footnotetext{
Received March 5, 2007; revised May 10, 2007; accepted May 28, 2007.

This work was supported by the 21st Century Center of Excellence Program (D-2 to Kyoto University); the Ministry of Education, Culture, Sports, Science, and Technology, Japan; and the Ministry of Education, Science, Sports and Culture [Grant-in-Aid for Scientific Research (C), 19500290], Japan. N.H. was supported by the Japan Society for the Promotion of Science (17-2087). We are grateful to Dr. Ryota Kanai and Neil S. Halelamien for their valuable comments on this manuscript and also to Dr. Tomonori Kito for his technical assistance.

Correspondence should be addressed to Dr. Eiichi Naito, National Institute of Information and Communication Technology (ICT), Research Department 1, Kobe Advanced ICT Research Center, Biophysical ICT Group, ATR Computational Neuroscience Laboratories, 2-2-2 Hikaridai, Seika-cho, Soraku-gun, Kyoto 619-0288, Japan. E-mail: enaito@atr.jp.

D01:10.1523/JNEUROSCI.0970-07.2007

Copyright $\odot 2007$ Society for Neuroscience $\quad$ 0270-6474/07/277047-07\$15.00/0
}

and Cohen, 1998). This integration strategy of multisensory information allows us to perceive our limb position at a single spatial location, even when the visual and somatosensory systems provide independent, perhaps conflicting, information.

Previous studies have suggested that the frontoparietal cortices are activated when a coherent percept of limb position is formed based on multimodal information sources in humans (Ehrsson et al., 2004) and in nonhuman primates (Graziano, 1999; Graziano et al., 2000). In the present study, we introduced a discrepancy between visual and somatosensory information for hand position: the somatosensory system signals kinesthetic information that the hand is moving, while visual information indicates that the same hand is stationary. In this situation, the kinesthetic information continuously generates positional discrepancy from the visually stationary hand, and thus the brain has to continuously resolve the discrepancy. This approach allows us to focus on the neuronal process that leads to a coherent perception of our hand position and helps our understanding of how the brain resolves the multisensory conflict by relying on vision.

To provide a continuous kinesthetic stimulation, we took advantage of a kinesthetic illusion that is elicited by vibratory stimulation to the tendon of a limb muscle (Goodwin et al., 1972; Naito, 2004). In this illusion, the vibration of the tendon excites 
its muscle spindle afferents (Goodwin et al., 1972; Burke et al., 1976; Roll and Vedel, 1982; Gandevia, 1985; Roll et al., 1989), and the brain continuously receives and processes the kinesthetic inputs. With eyes closed, people experience a sensation of slow movement of their vibrated limbs in the absence of actual movement, intention to move, or sense of effort (Kito et al., 2006). If their motionless limb is viewed during the vibratory stimulation, their sensation of the illusory limb movement weakens (Lackner and Taublieb, 1984). This attenuation of perceived limb movement is a paradigm of visual dominance over kinesthesia.

In the present study, right-handed healthy participants are given the vibratory tendon stimulation either on the right or left immobile hand, while viewing an on-line video image of either the vibrated [ipsilateral (IPSI)] or nonvibrated [contralateral (CONTRA)] static hand. Cross-modal discrepancy as to the position of the same hand is present in the IPSI condition but not in the CONTRA condition. Thus, the attenuation of the illusory movement is expected predominantly in the IPSI condition, and the CONTRA condition serves as a control.

Next, we identify brain activity associated with the neuronal process for resolving the discrepancy of the hand position between vision and kinesthesia (IPSI). We expect activation of the posterior parietal cortex (PPC), because the PPC is often activated during tasks, such as the acquisition of novel visuomotor behaviors in a distorted visual space, that require the brain to resolve a sensory discrepancy between limb movement/positions sensed by vision and kinesthesia/proprioception (Clower et al., 1996; Inoue et al., 1997, 2000). We subsequently test these predictions in behavioral and functional magnetic resonance imaging (fMRI) experiments.

\section{Materials and Methods \\ Participants}

Twenty-two right-handed (Oldfield, 1971) healthy male (20) and female (2) volunteers (18-27 years of age) participated in the behavioral experiment. Eight healthy right-handed male volunteers (18-36 years of age) with no history of neurological or other disease participated in the fMRI experiment. All participants had given their written informed consent before the experiments, and the Ethical Committee of the National Institute of Physiological Science had approved the study. The fMRI experiment was performed following the principles and guidelines of the Declaration of Helsinki (1975).

\section{Behavioral experiment}

The participants received vibratory stimulation on the tendon of the wrist extensor carpi ulnaris (ECU) muscle to induce the illusory flexion movement of the left or the right vibrated hand, while viewing the live image of the vibrated hand (IPSI) or the contralateral (nonvibrated) hand (CONTRA) (Fig. 1a). In the control condition, they received the vibratory stimulation with their eyes closed (CLOSE).

The vibrator was nonmagnetic (Illusor; Umihira, Kyoto, Japan) and driven by constant air pressure provided by an air compressor (Air King GTAC 1525; Great Tool, Taipei, Taiwan) (Naito et al., 2007). The amplitude of the vibratory stimuli was approximately $\pm 3.5 \mathrm{~mm}$. We know from our previous study that the intensity of the illusion depends on vibratory frequency (Naito et al., 1999). Thus, we varied the vibration frequency from 30 to $90 \mathrm{~Hz}$, with a $10 \mathrm{~Hz}$ step to examine the visual effect on a range of illusion intensities. The seven frequencies were adjusted by
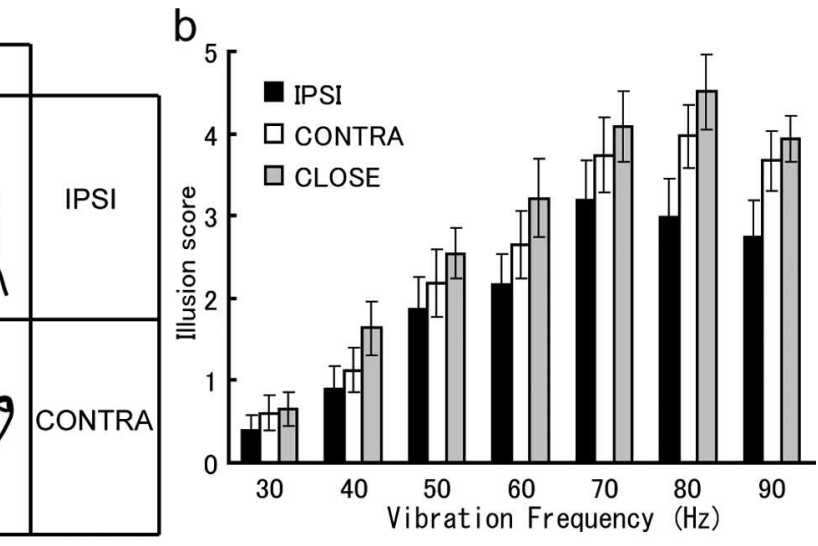

Figure 1. Conditions and results of the behavioral experiment. $\boldsymbol{a}$, The participants were given vibratory stimulation to their tendon, while viewing either the vibrated hand (IPSI) or the nonvibrated hand (CONTRA) through an on-line video camera. The (

changing the air pressure to the vibrator. One experimenter manually operated the vibrator by applying it to the skin with light pressure.

The live visual image of the participant's own hand was shown to the participants by a head-mounted display (Eye-Trek FMD-150W; Olympus, Tokyo, Japan) receiving the input from a video camera (SS2000CLM; Kyohritsu, Osaka, Japan). During the experiment, the participants lay comfortably on the bed in the supine position, with their arms extended in a relaxed manner, parallel to the trunk. Hands were semipronated and placed along the lateral surface of the body, but without contact $(\sim 20 \mathrm{~cm})$. The video camera was set above the hands, ensuring a radial view (Fig. 1a). The distance between the camera and the hand was $\sim 45 \mathrm{~cm}$, with the position and size of the hand image on the display consistent across all conditions. The image included the whole hand (from the wrist joint to the tips of fingers), and the body of the vibrator was not included in the image, because the vibration site was more proximally located than the hand image. Thus, the participants viewed live images of their hands as shown in Figure $1 a$. Before the experiment, each participant was requested to move his/her hand while viewing it through the display so that they could verify that the hands viewed on the display were theirs.

The seven frequencies were tested in each visual (IPSI, CONTRA, and CLOSE) condition for both left and right hands; thus, there were 21 conditions for each hand. To minimize the reduction of sensitivity of the muscle spindle receptors by tendon vibration repetition (Ribot-Ciscar and Roll, 1998), only one trial was assigned per condition. The order of each condition was pseudorandomized. The tendon was vibrated for $24 \mathrm{~s}$ on each trial. After each trial, the participants had to score the degree of perceived hand flexion by giving a score from 0 to 10 (illusion score). They had to give a score of 10 when they experienced illusory movements until the maximally flexed angle or a score of 0 when they felt no illusion (Naito et al., 1999). They were also required to replicate the experienced maximum angle of illusory flexion by actually flexing their wrists (Naito et al., 2002a). We measured this angle with a protractor.

For the statistical analysis on visual effects on the illusion, we performed three-factorial [visual conditions (IPSI, CONTRA, or CLOSE; 3) $\times$ vibratory frequencies $(30-90 \mathrm{~Hz} ; 10 \mathrm{~Hz}$ steps; 7$) \times$ hands (left or right hand; 2)] ANOVA (repeated measurement; $n=22$ ) both for the illusion score and for the replicated angle, separately. For the post hoc comparison, we used the $t$ test with Bonferroni correction for the number of comparisons.

\section{fMRI experiment}

Scanner. A 3.0 T Siemens (Erlangen, Germany) scanner (MAGNETOM Allegra), with head-coil provided T1-weighted anatomical images (3DSPGR $)$ and functional T2* ${ }^{*}$ weighted echoplanar images $(64 \times 64$ matrix; $3.0 \times 3.0 \mathrm{~mm}$; echo time, $30 \mathrm{~ms}$ ). One functional image volume of the 
brain was collected every $3 \mathrm{~s}$ (repetition time, $3000 \mathrm{~ms}$ ). A functional image volume comprised 44 slices of $3 \mathrm{~mm}$ thickness, which ensured that the whole brain was within the field of view $(192 \times 192 \mathrm{~mm})$.

Tasks. Eight participants rested comfortably in a supine position in the MR scanner. The postures of the forearms and hands were the same as those in the behavioral experiment. The participants were instructed to completely relax their bodies and not to make any movements during scanning.

The experiment was conducted with a 3 (somatosensory) $\times 2$ (visual) factorial design. We tested this design both for the left and right hands. As the somatosensory conditions, we vibrated either the tendon of the wrist extensor (ECU) muscles as in the behavioral experiment [tendon vibration condition $(\mathrm{T})]$ or the skin surface over the nearby bone just beside the tendon [i.e., the processus styloideus ulnae; bone vibration condition (B)]. We used the same nonmagnetic vibrator as that used in the behavioral experiment to apply identical vibratory stimulation. One condition (T) elicits illusory hand flexion movements as in the behavioral experiment, whereas the other condition (B) only elicits sensation of skin vibration with no reliable illusion (Naito and Ehrsson, 2006). Hence, this B condition serves as a somatosensory control for the $\mathrm{T}$ condition to depict purely the brain activity related to the kinesthetic experience. The contact surface on the skin was $\sim 1 \mathrm{~cm}^{2}$ for both vibratory ( $\mathrm{T}$ and $\mathrm{B}$ ) conditions. We also included a condition in which no vibratory stimuli were provided $(\mathrm{N})$. In all conditions, the participant's hand was immobilized by a wrist brace. This was done to ensure that the participants' hands did not move during the illusory experience $(\mathrm{T})$ and to prevent the vibrated hand from moving by the slight pressure of the vibrator applied on the wrist $(\mathrm{T}$ or B). In the behavioral experiment, we found that the visual effect on the illusion was highest at $80 \mathrm{~Hz}$ (see Results). Therefore, we only adopted this frequency for the fMRI experiment to obtain the maximum effect.

For the visual conditions, the participants viewed a live image of the vibrated hand (IPSI) or the contralateral (nonvibrated) hand (CONTRA). These visual images were presented through the same video camera (MR compatible) as used in the behavioral experiment. We took special care that position of the camera and view of their hands were quite similar to the situation in the behavioral experiment (see above and Fig. 1a). Before the scan, we put a mark on the radial side of the wrist joint, and the participants were requested to fixate on this point throughout the scanning to restrict eye movements. Their hands were set straight and approximately centered in the participant's view. On the scanner, they viewed the visual images through a mirror located just in front of their eyes, and the images were projected from outside of the scanner room. Before the scanning, we ensured that the participants could see their entire hand in the image and also that the body of the vibrator was not in the image (see above).

One experimenter in the scanner room manually operated the vibrator by applying it to the skin with light pressure. The instructions regarding the stimulus conditions as well as the onset and offset timings of the vibration were given to the experimenter by computer-generated visual cues projected onto the white surface of the scanner (the participants in the scanner could not see this visual information).

Each participant had eight sessions: in four of those sessions, the right hand was vibrated, and in the other four, the left hand was vibrated. Leftand right-hand sessions were alternated. For each hand, we assigned two sessions for the IPSI condition and two for the CONTRA condition. Each session included three conditions $(\mathrm{T}, \mathrm{B}$, and $\mathrm{N}$ ) and was repeated three times. Each condition lasted for $24 \mathrm{~s}$ (eight functional images). The order of conditions was randomized according to a balanced schedule. A period of $6 \mathrm{~s}$ before the start of each condition was defined as a condition of no interest and was used to set the vibrator positioned to the site of vibration. In each session, we collected 90 functional volumes, and a total of $8 \times 90$ volumes were collected per participant.

During the scanning, the participants were requested to be aware of the sensation from the vibrated hand under each condition. After each session, they were asked whether they felt an illusion during tendon vibration $(\mathrm{T})$, and they scored the illusion as in the behavioral experiment.

\section{Data analysis}

Analysis of the behavioral data in the fMRI experiment. We first calculated the mean value of the illusion scores for each condition (IPSI and CONTRA) per participant and performed a two-factorial [visual conditions (IPSI or CONTRA; 2$) \times$ hand (left or right hand; 2 ) ] ANOVA (repeated measurement; $n=8$ ) on the mean values.

fMRI data analysis. The fMRI data were analyzed with the Statistical Parametric Mapping software (SPM99; http://www.fil.ion.ucl.ac.uk/ spm; Wellcome Department of Cognitive Neurology, London, UK). The functional images were realigned to correct for head movements, coregistered with each participant's anatomical MRI, and transformed (linear and nonlinear transformation) to the format of the Montréal Neurological Institute standard brain. The functional images were scaled to 100 , spatially smoothed with an $8 \mathrm{~mm}$ full-width at half-maximum (FWHM) isotropic Gaussian kernel, and smoothed in time by a $4 \mathrm{~s}$ FWHM Gaussian kernel. A linear regression model (general linear model) was fitted to the pooled data from all participants to increase the sensitivity of the analysis [fixed-effect model; as in Naito and Ehrsson (2006)]. The validity of this approach, in terms of consistency of effects across all participants in the group, was confirmed by single-subject analyses (see below). Each condition was modeled with a boxcar function delayed by $4 \mathrm{~s}$ and convoluted with the standard SPM99 hemodynamic response function. Because our behavioral experiment indicates that the illusion starts a few seconds after the vibration onset, we modeled the period of the first $3 \mathrm{~s}$ of all experimental conditions as an effect of no interest.

Brain activations during IPSI condition. We defined a linear contrast in the general linear model to identify activity that was exclusively related to the IPSI-T condition. Because no behavioral difference was observed between the left and right hands in the fMRI experiment (see Results), we pooled the data from the left and right hands and examined the interaction between the site of vibration (T or B) and vision (IPSI or CONTRA) in a $2 \times 2$ factorial design [(IPSI-T vs IPSI-B) vs (CONTRA-T vs CONTRA-B)]. We used a contrast of IPSI-T versus IPSI-B as an inclusive mask ( $p<0.05$ uncorrected) to ensure that only the voxels showing activation from the corresponding control condition were included. The rationale of this design was to ensure that the resultant interaction term is related only with the interaction of visual and kinesthetic information for the same hand (IPSI-T) and is not attributable to possible confounding variables, such as simple differences in tendon vibration, cutaneous vibrotactile stimulation, or visual images of the hand. A voxelwise threshold of $p<0.001$ uncorrected $(t>3.09)$ was used to generate the cluster images. For the statistical inference, we used a threshold of $p<0.05$ or better at the cluster level after a correction for multiple comparisons in the whole brain space.

To further confirm that the areas revealed in this analysis are consistently relevant to the left and right hands, we performed a conjunction analysis (right [(IPSI-T vs IPSI-B) vs (CONTRA-T vs CONTRA-B)] $\cap$ left [(IPSI-T vs IPSI-B) vs (CONTRA-T vs CONTRA-B)] $)(p<0.05$ corrected) (Price and Friston, 1997; Friston et al., 2005).

Single-subject analyses. The statistical analysis was based on the functional data pooled across participants using a fixed-effect analysis $(p<$ 0.05 corrected), in which the results may be biased by a minority of participants showing strong effects. To make sure that the group results were representative for all of the eight participants, we analyzed the individual data (Naito and Ehrsson, 2006). All image-processing steps were identical to those used in the group analysis (see above). The same general linear model as in the group analysis was used, with the only difference being that we considered the functional data from each participant separately. We made a linear contrast of (IPSI-T vs IPSI-B) versus (CONTRA-T vs CONTRA-B) in each participant and then probed for increases of the blood oxygenation level-dependent (BOLD) signal ( $p<$ 0.05 uncorrected) in a volume of radius $16 \mathrm{~mm}$ around peaks of activations detected in the group analysis. We report the number of participants that exhibited a BOLD signal increase in the relevant areas.

Correlation analysis. The PPC was activated in the IPSI-T condition, in which robust visual dominance over kinesthetic illusion was observed (see Results, Fig. 2b). We performed a correlation analysis to test whether activity in the PPC cluster reflects the degree of kinesthetic attenuation caused by vision. 
First, we calculated the mean of the illusion scores obtained from six T epochs in the two IPSI sessions. This was performed for each participant, and each hand was treated separately. The same procedure was performed for the CONTRA sessions, and then we calculated the difference in the mean scores between the IPSI-T and CONTRA-T conditions. This difference indicates the degree of visual dominance over kinesthetic illusion (i.e., degree of kinesthetic attenuation) in the IPSI condition with the CONTRA condition as the baseline. We used those values, which we refer to as the visual attenuation index, as a covariate in the correlation analysis.

Second, for the fMRI data, we defined a linear contrast in the general linear model to identify activations in the contrast of (IPSI-T vs IPSI-B) versus (CONTRA-T vs CONTRA-B). This was done for each participant, and a separate contrast was defined for each hand. From the individual contrasts, we calculated the contrast parameter estimates related to the visual effect in the illusion in the IPSI condition compared with that in the CONTRA condition.

Next, we tested whether the parameter estimates of the PPC voxels correlate with the visual attenuation index by using a simple regression analysis (14 degrees of freedom; we pooled the data from the left and right hands). A search volume was set in the PPC cluster obtained in the group analysis (see above and Fig. $2 b$ ).

\section{Results}

\section{Behavioral experiments}

During tendon vibration, all participants experienced kinesthetic illusory hand flexion, but they reported that its intensity was dependent on the vibratory frequency and the type of visual information (IPSI, CONTRA, or CLOSE). The results are summarized in Figure $1 b$. The illusion was strongest when the tendon was vibrated in the range of $70-80 \mathrm{~Hz}$ under all visual conditions. The visual image of the contralateral nonvibrated hand (CONTRA) seems to slightly attenuate the illusion compared with that of the CLOSE condition, but the attenuation was robust when the participants viewed a live image of the vibrated hand (IPSI). The attenuation was greatest at $80 \mathrm{~Hz}$ in the IPSI condition. No overt hand movements appeared in any of the trials.

A three-factorial [visual conditions $(3) \times$ vibratory frequencies (7) $\times$ hands (2)] ANOVA of illusion scores revealed significant main effects of the visual conditions $\left(F_{(2,42)}=11.2 ; p<\right.$ $0.001)$ and the vibratory frequencies $\left(F_{(6,126)}=49.2 ; p<0.001\right)$ (Fig. 1b). There was no significant difference between the left and right hands, and no significant interaction was observed. A post hoc analysis revealed that the attenuation of the illusion was significantly stronger in the IPSI condition (mean illusion score = 2.0 ) than both the CONTRA condition (mean illusion score $=$ 2.6 ; $\mathrm{df}=21 ; t=3.2 ; p<0.05$ corrected) and the CLOSE condition (mean illusion score $=2.9 ; \mathrm{df}=21 ; t=4.0 ; p<0.005$ corrected). However, no significant difference was observed between the CONTRA and CLOSE conditions. When we compared the illusion scores between the IPSI and CONTRA conditions for each vibratory frequency, a significant difference was found only at $80 \mathrm{~Hz}$ (mean illusion score $=3.0$ for IPSI condition and 4.0 for CONTRA condition; $\mathrm{df}=21 ; t=3.0 ; p<0.05$ corrected).

When the same analysis was performed using the replicated angles as dependent variables, a similar pattern of results was also obtained (see supplemental material and supplemental Fig. 1a,b, available at www.jneurosci.org).

\section{fMRI experiment}

All participants reported that they experienced an illusion when the tendon was vibrated (T), but no reliable illusion was produced when the skin surface over the nearby bone was vibrated (B). A two-factorial [visual conditions (2) $\times$ hands (2)] ANOVA on the illusion scores revealed that the illusion was significantly
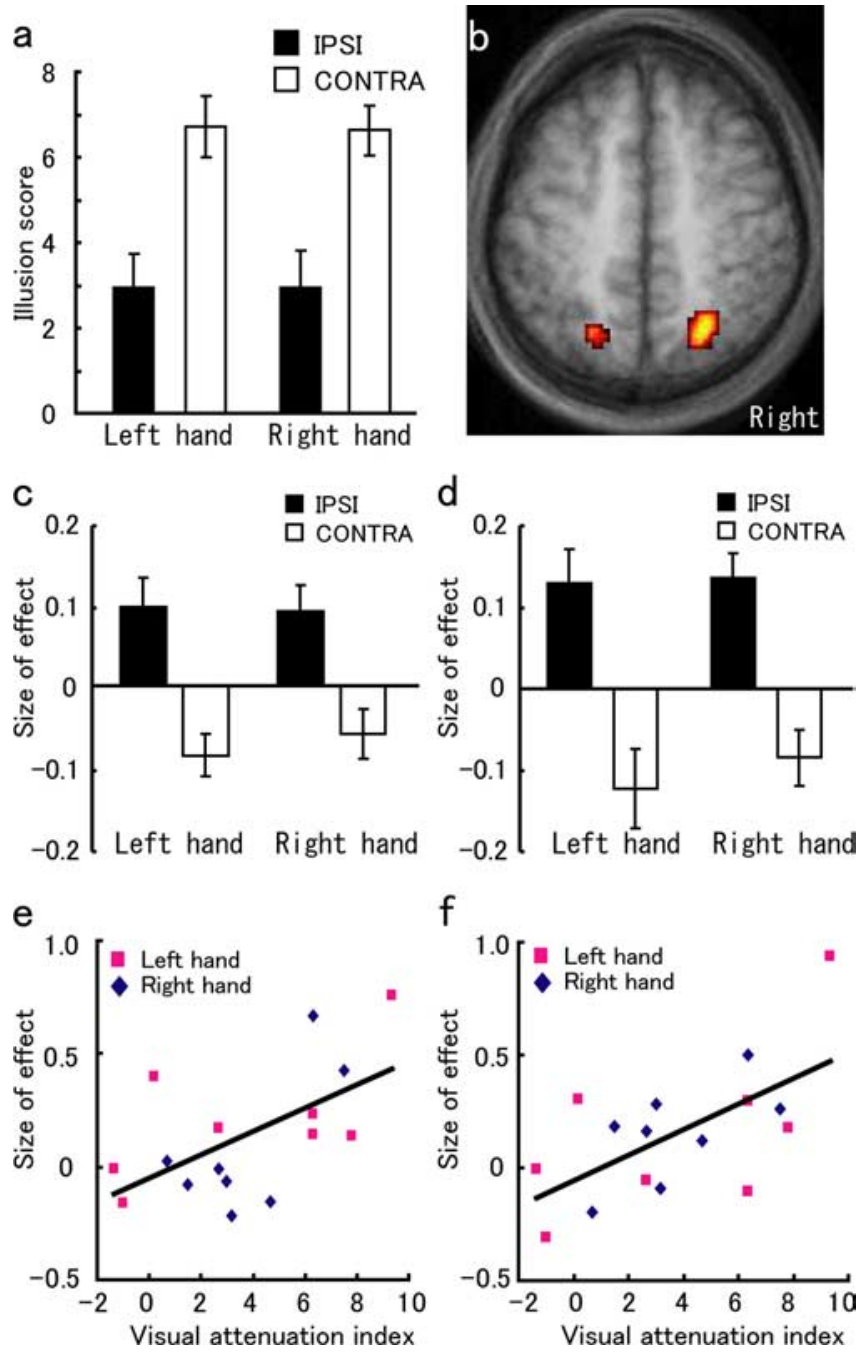

Figure 2. Results of the fMRI experiment. $\boldsymbol{a}$, The mean illusion scores during the fMRI experiment. $\boldsymbol{b}$, Activation of the PPC specific to the IPSI condition ( $p<0.001$ uncorrected for display purposes). A horizontal plane at $z=57$ is displayed. $\boldsymbol{c}, \boldsymbol{d}$, Activity (size of effect) of the PPC ( $\boldsymbol{c}$, left PPC; $-24,-69,57 ; \boldsymbol{d}$, right PPC; $27,-69,63)$ during the illusion under the IPSI and CONTRA conditions compared with the corresponding bone vibration condition. $\boldsymbol{e}, \boldsymbol{f}$, Correlation of the PPC activity with the visual attenuation index (see Materials and Methods) ( $\boldsymbol{e}$, left PPC; $-24,-66,63 ; \boldsymbol{f}$, right PPC, 24, $-66,66)$. The error bars indicate 1 SEM across participants $(n=8)$.

attenuated in the IPSI-T condition (mean illusion score $=2.9$ ) compared with the CONTRA-T condition (mean illusion score $=6.7)$, replicating the results of the behavioral experiment $\left[F_{(1,7)}=11.3 ; p<0.05\right.$ (Fig. $\left.\left.2 a\right)\right]$. Also as in the behavioral experiment, there was no significant difference between the leftand right-hand conditions and no significant interaction between the factors.

An analysis designed to identify the brain regions involved exclusively in the IPSI-T condition [(IPSI-T vs IPSI-B) vs (CONTRA-T vs CONTRA-B)] revealed activation only in the right PPC in the entire brain [peak coordinates $(x, y, z)=(27$, $-66,60) ; t=5.2 ; p<0.05$ corrected] (Fig. 2b,d). The cluster was mainly located in the posterior part of the superior parietal lobule (SPL) and extended laterally to the caudomedial part of the intraparietal sulcus. In the corresponding region of the left PPC, we also found a trend of increasing activity [peak, $(-24,-69,57)$; $t=3.9 ; p<0.001$ uncorrected; number of voxels $=19$ ] (Fig. $2 b, c)$. Single-subject analyses revealed that in all eight partici- 
Table 1. The coordinates of peaks of the PPC in individual participants [(IPSI-T vs IPSI-B) vs (CONTRA-T vs (ONTRA-B)]

\begin{tabular}{|c|c|c|c|c|c|c|c|c|}
\hline \multirow[b]{2}{*}{ Participants } & \multicolumn{4}{|c|}{ Right PPC } & \multicolumn{4}{|c|}{ Left PPC } \\
\hline & $x$ & $y$ & $z$ & $t$ values & $x$ & $y$ & $z$ & $t$ values \\
\hline 1 & 27 & -72 & 57 & 2.8 & n.s. & & & \\
\hline 2 & 24 & -69 & 63 & 4.1 & -24 & -63 & 63 & 3.4 \\
\hline 3 & 30 & -66 & 51 & 4.5 & -18 & -66 & 51 & 4.8 \\
\hline 4 & 30 & -63 & 57 & 5.6 & -24 & -63 & 57 & 4.3 \\
\hline 5 & 21 & -69 & 57 & 3.5 & -24 & -75 & 57 & 4.5 \\
\hline 6 & 27 & -60 & 69 & 1.7 & n.s. & & & \\
\hline 7 & 33 & -72 & 63 & 2.2 & -24 & -63 & 45 & 1.7 \\
\hline 8 & 18 & -60 & 57 & 1.7 & n.s. & & & \\
\hline
\end{tabular}

n.s., Not significant.

pants, activity significantly increased in the right PPC, and for five of eight participants, the increase was also observed in the left PPC (Table 1).

To further confirm that the activity of the PPC in the IPSI-T condition is consistently relevant to the left and right hands, we performed a conjunction analysis (see Materials and Methods). We found significant activation in the right PPC [peak, $(27,-69$, $63) ; t=3.4 ; p<0.05$ corrected] and also a trend of increased activity in the left PPC [peak, $(-24,-69,57) ; t=2.6 ; p<0.001$ uncorrected]. Finally, no significant difference was found in the interaction effects between the left and right hands [comparison of (IPSI-T vs IPSI-B) vs (CONTRA-T vs CONTRA-B) between left and right hands], suggesting that there was no systematic contralateral effect depending on the sides of hand in the present PPC activations. Hence, the PPC was bilaterally activated exclusively during the IPSI-T condition regardless of left- and righthand vibration, albeit with only a statistical trend of activation in the left PPC (Fig. 2c,d).

\section{Correlation analysis}

Attenuation of illusory hand movement was predominantly observed in the IPSI-T condition, in which the PPC was selectively activated. To further establish a relationship between PPC activation and the attenuation of the illusion, we tested whether the activity of the PPC region correlated with the degree of attenuation, as quantified as the visual attenuation index (see Materials and Methods). The results of this analysis demonstrated that the PPC regions identified by the interaction term correlate with the individual attenuation of the illusion [right peak, $(24,-66,66)$; $\mathrm{df}=14 ; r=0.62 ; p<0.005$ one-tailed; left peak, $(-24,-66,63)$; $\mathrm{df}=14 ; r=0.59 ; p<0.01$ one-tailed (Fig. $2 e, f)$ ]. This quantitative relationship between attenuation and the PPC activation corroborates the idea that the PPC is involved in the visual effect that attenuates the illusory percept.

\section{Discussion}

The behavioral experiment showed that visual information of a static hand attenuated the illusory kinesthetic hand movement when it was from the same hand as the kinesthetically stimulated one (IPSI). The fMRI study showed that the PPC is selectively activated during the attenuation of the illusion and that its activity level was correlated with the degree of kinesthetic attenuation caused by vision. These results suggest that the human PPC is involved in the multisensory processing between the visual and kinesthetic information and mediates the visual dominance over kinesthesia.
How the brain estimates limb positions when visual and kinesthetic information are simultaneously provided

During the IPSI condition, the visual information indicated that the hand position was stationary, whereas the kinesthesia (tendon vibration) indicated that the same hand was moving. Thus, the two sensory systems simultaneously provided discrepant information about the spatial location of that hand. Therefore, during tendon vibration, the brain had to somehow keep resolving the discrepancy so as to estimate the current location of that hand. This kind of computation is unnecessary during the CONTRA condition, in which the visual and kinesthetic information came from the two different hands. This may be why the attenuation of the illusion was robustly observed in the IPSI condition.

In general, the hand location specified by vision is more precise than that sensed by kinesthesia (see Introduction). This is also probably the case for the vision under the IPSI condition, because the participants knew that they were viewing their own hand, which was immobile throughout the tendon vibration. For the kinesthesia, they experienced various intensities of the illusion depending on the vibratory frequency. In addition, even when identical vibratory stimuli are applied to an identical tendon site, we know from our previous study that the intensity of illusion varies from trial to trial (Naito et al., 2002a). All of these support the idea that the accuracy of kinesthesia is poor compared with that of vision as regards to the ability to specify the absolute position of a limb. Hence, in the IPSI condition, the brain relied on vision rather than kinesthesia (Smeets et al., 2006), and the visual information about the static (vibrated) hand attenuated the kinesthetic sensation.

We obtained the largest attenuation of the illusion at $80 \mathrm{~Hz}$ (Fig. 1b), which is the frequency that elicited the strongest illusion when the eyes were closed (CLOSE) (Fig. 1b) (see also Naito et al., 1999). Because the vibratory stimulation induces the strongest percept of a hand movement at this frequency, the discrepancy between the visual and kinesthetic information as to the hand position also becomes largest. Therefore, the amount of discrepancy that brain has to resolve is largest at this frequency. A computational model suggests that when visual and kinesthetic information are simultaneously provided, the brain estimates the spatial location of a limb separately for vision and kinesthesia and determines the limb's location as a weighted (weight based on the precision of each information about limb position) average of the two estimates (Smeets et al., 2006). Because the precision of visual information is higher, the kinesthetic estimate drifts toward the visual one. Thus, we conjecture that the kinesthetic estimate would drift toward the visual estimate to the greatest degree at 80 $\mathrm{Hz}$. The brain activation exclusively related to the IPSI-T condition might participate in the specific neuronal process for achieving this type of neuronal computation (see below).

\section{Activation in the PPC during the IPSI-T condition}

The PPC (posterior part of SPL) activation seems to be specific to the neuronal computation associated with the cancellation of the illusory hand movement while viewing the vibrated static hand (IPSI-T). First of all, in our factorial design [(IPSI-T vs IPSI-B) vs (CONTRA-T vs CONTRA-B)], effects related to the kinesthetic inputs (T), skin vibration (B), and different types of visual images 
are all counterbalanced, and thus none of these factors as such are responsible for the posterior SPL activation. Moreover, the present SPL activation (Fig. $2 b$ ) cannot be attributed to the illusory percept of hand movement per se, because it has never been identified in the series of our previous studies (Naito et al., 1999, 2002a,b, 2005, 2007) in which right-handed blindfolded participants merely experienced the kinesthetic illusion (unimodal situation). Also, the parietal region was not activated as the main effect of tendon vibration (see supplemental material and supplemental Fig. 2, available at www.jneurosci.org), nor during the illusion when the participants viewed the contralateral nonvibrated hand (CONTRA) (Fig. $2 c, d$ ).

Although the illusory movement was attenuated in the IPSI-T condition, it was not completely eliminated (that is, the discrepancy between vision and kinesthesia continued to exist during the tendon stimulation). Together with the finding that the activity in the posterior SPL was correlated with the degree of visual effect that attenuates kinesthesia (Fig. 2e,f), the strong SPL activation in the IPSI-T condition is likely to reflect the process for resolving the positional discrepancy in the situation in which the vision dominates kinesthesia [conversely, the kinesthetic attenuation per se may be demonstrated as the reduction of precentral gyrus activity, which is suggested to reflect the somatic perception of limb movement (Naito et al., 2002b) (see also supplemental material and supplemental Fig. 3, available at www.jneurosci.org)].

The bilateral parietal activations related to this process (Fig. $2 b$ ) could be a result of the instruction to participants to centrally fixate the visual hand image. Neurons in the caudal part of the SPL (PEc; see below) in monkeys have visual properties with a wide range of receptive fields that may cover both the ipsilateral and contralateral hemifield (Squatrito et al., 2001). Thus, the present visual stimuli are likely to have activated both the left and right hemispheres.

Recent quantitative receptor autoradiography studies have revealed that the human SPL can be subdivided into different regions, [i.e., anterior (area 5 and anterior area 7) and posterior (posterior area 7) (Scheperjans et al., 2005a,b)]. The present SPL activations (Fig. $2 b$ ) seem to be located in the posterior part of the human SPL. The posterior part has receptor distributions similar to those of the visual cortices, suggesting that neurons in this parietal region may have visually predominant properties (Scheperjans et al., 2005b). Therefore, the present posterior parietal activation is a location suitable for visually driven suppression of kinesthetic information. In monkeys, area PEc in the posterior part of the SPL (Scheperjans et al., 2005b) responds to visual stimuli (Squatrito et al., 2001), but to some extent, it responds also to somatosensory stimuli such as passive joint rotation (Breveglieri et al., 2006). Given this, it is conceivable that the posterior part of the SPL in humans also processes both visual and kinesthetic information for allocating the spatial position of a hand.

Indeed, neuronal activity in this region during a reaching movement can be modulated when the visual feedback of the hand is present, in nonhuman primates (area PEc) (BattagliaMayer et al., 2001) and also in humans (posterior part of SPL) (Kertzman et al., 1997; Grefkes et al., 2004). Likewise, the similar region is also activated during hand-writing tasks that require on-line correction of trajectories of hand movements by vision (Seitz et al., 1997; Siebner et al., 2001). These studies support the idea that the parietal cortex has a role in adjusting the perceived spatial location of the hand in the visual space, potentially also contributing to accurate motor control (Battaglia-Mayer et al., 2000, 2001).
Our finding is also consistent with neurological studies on patients with a PPC (including SPL) lesion. The PPC patients exhibit impairment in judging whether hand movements they viewed are derived from their own hands (Sirigu et al., 1999). This could be accounted for as a deficit in achieving perceptual coherence for the spatial location of the hand in a situation in which the brain needs to combine kinesthetic and visual information. Likewise, reaching to a visual target becomes inaccurate for patients with SPL lesions (optic ataxia) (Bálint, 1909; Battaglia-Mayer and Caminiti, 2002). This could be also explained by a deficit in precise allocation of hand position in the visual space. Together, these neurological findings show that the parietal region mediates visuokinesthetic multisensory processing for perception of hand position.

Finally, we can relate our finding to the previous studies investigating the multimodal coherent perception of limb position. When participants view a rubber hand being stroked, while their own unseen hand (set in a location separate from the rubber hand) is synchronously stroked, after a few seconds, the location of their own hand is perceived as being at the same position as that of the rubber hand (rubber hand illusion) (Botvinick and Cohen, 1998). This coherent percept between the two sensory modalities was most strongly correlated with the activity in the ventral premotor cortex (Ehrsson et al., 2004). However, during the period after the initiation of stroking, but before the onset of the illusion, a broader brain network including the PPC (caudal part of intraparietal sulcus) was activated (Ehrsson et al., 2004). This activation is likely associated with brain processes to create this illusion using multisensory information. The common denominator between the present and the previous findings is that the parietal activation is specific to the period in which the brain resolves a discrepancy between limb positions sensed by vision and kinesthesia, and the visual dominance over kinesthesia takes place subsequently. Thus, the PPC activation is tightly related to the creation of a coherent perception of a limb position by way of visual dominance over kinesthesia.

In conclusion, the computation to estimate hand position based on visual information (visual dominance over kinesthesia) takes place in the PPC. This neuronal computation could be a prerequisite for maintaining perceptual coherence of our body image (Haggard and Wolpert, 2005) when the brain receives information regarding our body parts from multiple sensory modalities.

\section{References}

Bálint R (1909) Seelenlähmung des 'schauens', optische ataxie, räumliche störung der aufmerksamkeit. Mschr Psychiat Neurol 25:51-81.

Battaglia-Mayer A, Caminiti R (2002) Optic ataxia as a result of the breakdown of the global tuning fields of parietal neurones. Brain 25:225-237.

Battaglia-Mayer A, Ferraina S, Mitsuda T, Marconi B, Genovesio A, Onorati P, Lacquaniti F, Caminiti R (2000) Early coding of reaching in the parietooccipital cortex. J Neurophysiol 83:2374-2391.

Battaglia-Mayer A, Ferraina S, Genovesio A, Marconi B, Squatrito S, Molinari M, Lacquaniti F, Caminiti R (2001) Eye-hand coordination during reaching. II. An analysis of the relationships between visuomanual signals in parietal cortex and parieto-frontal association projections. Cereb Cortex 11:528-544.

Botvinick M, Cohen J (1998) Rubber hands "feel" touch that eyes see. Nature 391:756.

Breveglieri R, Galletti C, Gamberini M, Passarelli L, Fattori P (2006) Somatosensory cells in area PEc of macaque posterior parietal cortex. J Neurosci 26:3679-3684.

Burgess PR, Wei JY, Clark FJ, Simon J (1982) Signaling of kinesthetic information by peripheral sensory receptors. Annu Rev Neurosci 5:171-187.

Burke D, Hagbarth K, Lofstedt L, Wallin G (1976) The responses of human 
muscle spindle endings to vibration of non-contracting muscles. J Physiol (Lond) 261:673-693.

Clower DM, Hoffman JM, Votaw JR, Faber TL, Woods RP, Alexander GE (1996) Role of posterior parietal cortex in the recalibration of visually guided reaching. Nature 383:618-621.

Ehrsson HH, Spence C, Passingham RE (2004) That's my hand! Activity in premotor cortex reflects feeling of ownership of a limb. Science 305:875-877.

Friston KJ, Penny WD, Glaser DE (2005) Conjunction revisited. NeuroImage 25:661-667.

Gandevia SC (1985) Illusory movements produced by electrical stimulation of low-threshold muscle afferents from the hand. Brain 108:965-981.

Goodwin GM, McCloskey DI, Matthews PBC (1972) Proprioceptive illusions induced by muscle vibration: contribution by muscle spindles to perception? Science 175:1382-1384.

Graziano MS (1999) Where is my arm? The relative role of vision and proprioception in the neuronal representation of limb position. Proc Natl Acad Sci USA 96:10418-10421.

Graziano MSA, Gross J (1998) Spatial maps for the control of movement. Curr Opin Neurobiol 8:195-201.

Graziano MSA, Cooke DF, Taylor CSR (2000) Coding the location of the arm by sight. Science 290:1782-1786.

Grefkes C, Ritzl A, Zilles K, Fink GR (2004) Human medial intraparietal cortex subserves visuomotor coordinate transformation. NeuroImage 23:1494-1506.

Haggard P, Wolpert DM (2005) Disorders of body schema. In: High-order motor disorders: from neuroanatomy and neurobiology to clinical neurology, Chap 14 (Freund H-J, Jeannerod M, Hallett M, Leiguarda R, eds), pp 261-271. Oxford: Oxford UP.

Head H, Holmes G (1911) Sensory disturbances from cerebral lesions. Brain 34:102-254.

Inoue K, Kawashima R, Satoh K, Kinomura S, Goto R, Sugiura M, Ito M, Fukuda H (1997) Activity in the parietal area during visuomotor learning with optical rotation. NeuroReport 8:3979-3983.

Inoue K, Kawashima R, Satoh K, Kinomura S, Sugiura M, Goto R, Ito M, Fukuda (2000) A PET study of visuomotor learning under optical rotation. NeuroImage 11:505-516.

Kertzman C, Schwarz U, Zeffiro TA, Hallett M (1997) The role of posterior parietal cortex in visually guided reaching movements in humans. Exp Brain Res 114:170-183.

Kito T, Hashimoto T, Yoneda T, Katamoto S, Naito E (2006) Sensory processing during kinesthetic aftereffect following illusory hand movement elicited by tendon vibration. Brain Res 1114:75-84.

Lackner JR, Taublieb AB (1984) Influence of vision on vibration-induced illusions of limb movement. Exp Neurol 85:97-106.

Naito E (2004) Sensing limb movements in the motor cortex: how humans sense limb movement. Neuroscientist 10:73-82.

Naito E, Ehrsson HH (2006) Somatic sensation of hand-object interactive movement is associated with activity in the left inferior parietal cortex. J Neurosci 26: 3783-3790.

Naito E, Ehrsson HH, Geyer S, Zilles K, Roland PE (1999) Illusory arm movements activate cortical motor areas: a PET study. J Neurosci 19:6134-6144

Naito E, Kochiyama T, Kitada R, Nakamura S, Matsumura M, Yonekura Y,
Sadato N (2002a) Internally simulated movement sensations during motor imagery activate the cortical motor areas and the cerebellum. J Neurosci 22:3683-3691.

Naito E, Roland PE, Ehrsson HH (2002b) I feel my hand moving: a new role of the primary motor cortex in somatic perception of limb movement. Neuron 36:979-988.

Naito E, Roland PE, Grefkes C, Choi HJ, EickhoffS, Geyer S, Zilles K, Ehrsson HH (2005) Dominance of the right hemisphere and role of area 2 in human kinesthesia. J Neurophysiol 93:1020-1034.

Naito E, Nakashima T, Kito T, Aramaki Y, Okada T, Sadato N (2007) Human limb-specific and non limb-specific brain representations during kinesthetic illusory movements of the upper and lower extremities. Eur J Neurosci 25:3476-3487.

Oldfield RC (1971) The assessment and analysis of handedness: the Edinburgh inventory. Neuropsychologia 9:97-113.

Price CJ, Friston KJ (1997) Cognitive conjunction: a new approach to brain activation experiments. NeuroImage 5:261-270.

Ribot-Ciscar E, Roll JP (1998) Ago-antagonist muscle spindle inputs contribute together to joint movement coding in man. Exp Brain Res 791:167-176.

Roll JP, Vedel JP (1982) Kinaesthetic role of muscle afferent in man, studied by tendon vibration and microneurography. Exp Brain Res 47:177-190.

Roll JP, Vedel JP, Ribot E (1989) Alteration of proprioceptive messages induced by tendon vibration in man: a microneurographic study. Exp Brain Res 76:213-222.

Scheperjans F, Grefkes C, Palomero-Gallagher N, Schleicher A, Zilles K (2005a) Subdivisions of human parietal area 5 revealed by quantitative receptor autoradiography: a parietal region between motor, somatosensory, and cingulate cortical areas. NeuroImage 25:975-992.

Scheperjans F, Palomero-Gallagher N, Grefkes C, Schleicher A, Zilles K (2005b) Transmitter receptors reveal segregation of cortical areas in the human superior parietal cortex: relations to visual and somatosensory regions. NeuroImage 28:362-379.

Seitz RJ, Canavan AG, Yaguez L, Herzog H, Tellmann L, Knorr U, Huang Y, Homberg V (1997) Representations of graphomotor trajectories in the human parietal cortex: evidence for controlled processing and automatic performance. Eur J Neurosci 9:378-389.

Siebner HR, Limmer C, Peinemann A, Bartenstein P, Drzezga A, Conrad B (2001) Brain correlates of fast and slow handwriting in humans: a PETperformance correlation analysis. Eur J Neurosci 14:726-736.

Sirigu A, Daprati E, Pradat-Diehl P, Franck N, Jeannerod M (1999) Perception of self-generated movement following left parietal lesion. Brain 122:1867-1874.

Smeets JB, van den Dobbelsteen JJ, de Grave DD, van Beers RJ, Brenner E (2006) Sensory integration does not lead to sensory calibration. Proc Natl Acad Sci USA 103:18781-18786.

Squatrito S, Raffi M, Maioli MG, Battaglia-Mayer A (2001) Visual motion responses of neurons in the caudal area pe of macaque monkeys. J Neurosci 21: $\mathrm{RC130(1-5).}$

van Beers RJ, Sittig AC, Denier van der Gon JJ (1996) How humans combine simultaneous proprioceptive and visual position information. Exp Brain Res 111:253-261.

van Beers RJ, Sittig AC, Denier van der Gon JJ (1998) The precision of proprioceptive position sense. Exp Brain Res 122:367-377. 